\title{
Effectiveness, Safety, and Patient Satisfaction of Liraglutide in Type 2 Diabetic Patients
}

Rafia Zameer ${ }^{1}$, Matiullah Kamin ${ }^{2}$, Umar Raja ${ }^{2}$, Muhammad Umar Wahab ${ }^{3,4}$, Osama Ishtiaq ${ }^{2}$, Kashif Raashid $^{2}$, Naveed Ahmed ${ }^{1}$, Asim.ur. Rehman ${ }^{1}$

1. Pharmacy, Quaid-I-Azam University, Islamabad, PAK 2. Endocrinology, Shifa International Hospital, Islamabad, PAK 3. Endocrinology, Umar Diabetes and Footcare Clinic, Islamabad, PAK 4. Diabetology, Umar Diabetes Foundation, Islamabad, PAK

Corresponding author: Matiullah Kamin, matiullah_shifa@yahoo.com

\section{Abstract \\ Background and Objectives}

Liraglutide, an analog of human glucagon-like peptide 1 (GLP-1), has been approved for the treatment of type 2 diabetes mellitus in Pakistan since 2016. It is a GLP-1 receptor agonist that has shown promising results in terms of not only glycemic control but also weight loss. Our study aimed to provide evidence regarding the safety and effectiveness of liraglutide in Pakistan and to look at the adherence rate and treatment satisfaction of patients using liraglutide.

\section{Methods}

This is an observational retrospective study that recruited patients who were treated with liraglutide. Data were collected at the first visit and follow-up. Morisky Green Levine Adherence Scale and Treatment Satisfaction Questionnaire for Medication (TSQM-9) were used for the determination of adherence and satisfaction with the treatment.

\section{Results}

A total of 70 patients were recruited in the study, The mean difference in weight, body mass index, glycated hemoglobin (HbA1C), systolic blood pressure, and diastolic blood pressure from baseline to follow-up was $5.36 \mathrm{~kg},-2.14 \mathrm{~kg} / \mathrm{m}^{2},-1.76 \%,-12.38 \mathrm{mmHg}$, and $5.55 \mathrm{mmHg}$, respectively. Nausea was the main side effect reported. TSQM-9 scores were compared from baseline, and it was found that patients are satisfied with the treatment and its effectiveness.

\section{Conclusions}

Our study has demonstrated the effectiveness of liraglutide as a monotherapy or combination therapy in the Pakistani population. Liraglutide led to reduction in HbA1C and weight. This is associated with high treatment satisfaction rate and adherence rate. Thus, liraglutide remains an effective though expensive treatment option in a country like Pakistan.

Received 07/24/2020

Review began $07 / 30 / 2020$ Review ended 08/10/2020 Published 08/22/2020

\section{() Copyright 2020}

Zameer et al. This is an open access article distributed under the terms of the Creative Commons Attribution License CC-BY 4.0., which permits unrestricted use, distribution, and reproduction in any medium, provided the original author and source are credited.
Categories: Endocrinology/Diabetes/Metabolism

Keywords: liraglutide, hemoglobin a1c, retrospective study, type 2 diabetes mellitus

\section{Introduction}

Type 2 diabetes mellitus (T2DM) is a complex metabolic disorder with multiple pathophysiological mechanisms [1]. With increase understanding of the pathophysiological mechanisms behind T2DM, there have been several new treatment options over the last decade or so. One such treatment option is glucagonlike peptide 1 (GLP-1) receptor agonists that have shown promising results in terms of not only glycemic control but also weight loss [2].

Injectable GLP-1 receptor agonist mimics endogenous GLP-1 by way of stimulating pancreatic insulin secretion as well as suppressing glucagon production [2]. Regardless of its function in glucose homeostasis, the GLP-1 receptors are widely distributed throughout the body, including the heart, brain, gastrointestinal tract, and pancreas. GLP-1 may exert its outcomes through both receptor-dependent and receptorindependent mechanisms and through the actions of both the intact peptide and its metabolites [3]. GLP-1 receptor agonists have shown cardiovascular benefits as well as a reduction in albuminuria independent of glycemic control, causing a new dimension within the control of T2DM [4].

Liraglutide, an analog of human GLP-1, has been approved for the treatment of T2DM in Pakistan since 2016 [5]. Although there are real-world data about the safety and effectiveness of liraglutide worldwide, there is a dearth of such data in South Asia. So far, there have been small-scale studies in India and Pakistan 
looking at only the safety and effectiveness of liraglutide [5,6]. Our study aimed not only to provide realworld evidence regarding the safety and effectiveness of liraglutide in the Pakistani population but also to look at adherence rate and treatment satisfaction of patients using liraglutide.

\section{Materials And Methods}

This is an observational retrospective study that recruited patients from endocrinology clinics of Shifa International Hospital Islamabad and Umar Diabetes and Foot Care Centre, Islamabad, Pakistan, from September 2018 to June 2019. The study was conducted after approval by the respective Ethical Review Committees. All patients with T2DM above 18 years with glycated hemoglobin (HbA1c) $\geqslant 7 \%$ and body mass index $(\mathrm{BMI}) \geqslant 25 \mathrm{~kg} / \mathrm{m}^{2}$ were prescribed liraglutide $0.6 \mathrm{mg}$ subcutaneously once a day irrespective of their meal at the same time initially for the first five days, and from the sixth day they were switched to a dosage of $1.2 \mathrm{mg}$ with further up-titration to $1.8 \mathrm{mg}$ based on tolerance. Other prescribed drugs were advised to be taken accordingly. Patients were excluded if they had type 1 diabetes and a glomerular filtration rate (GFR) < $45 \mathrm{~mL} / \mathrm{min} / 1.73 \mathrm{~m}^{2}$.

At first visit, baseline data were collected using a pre-designed data collection form, which included age, weight, BMI, blood pressure, and HbA1c. Morisky Green Levine (MGL) Adherence Scale and Treatment Satisfaction Questionnaire for Medication (TSQM-9) were used for the determination of adherence and satisfaction with the treatment [7,8]. Analysis was based on efficacy endpoints that were reduction in HbA1c, BMI, and fasting glucose levels from baseline values.

Statistical analyses were performed using IBM SPSS Statistics for Windows, Version 20.0 (IBM Corp., Armonk, NY, USA). Categorical variables were recorded as frequencies and percentages for each category and continuous variables were recorded as means and standard deviations. A paired t-test was applied for pre-post changes in clinical outcomes and treatment satisfaction, whereas analysis of variance (ANOVA) was applied to determine the differences between various diabetics groups.

\section{Results}

A total of 70 patients were recruited in the study, out of which $52.9 \%$ were males and $41.1 \%$ were females with a mean diabetes duration of $8.53 \pm 6.44$ years. Baseline characteristics are given in Table 1. Around $85 \%$ of the patients were taking $1.2 \mathrm{mg}$ of liraglutide once a day. In addition to liraglutide, patients were also reported to take metformin, sodium-glucose co-transporter-2 (SGLT2) inhibitors, sulfonylureas, and insulin. Most of the patients were moderately controlling their diet but were not exercising.

\begin{tabular}{|c|c|c|}
\hline S. No. & Variables & $\mathrm{n}(\%)$ \\
\hline 1 & Age (Years), Mean \pm SD & $49.35 \pm 10.91$ \\
\hline \multirow[t]{3}{*}{2} & Gender & \\
\hline & Male & $37(52.9)$ \\
\hline & Female & $33(47.1)$ \\
\hline 3 & Duration of Diabetes (Years), Mean \pm SD & $8.53 \pm 6.44$ \\
\hline \multirow[t]{8}{*}{4} & Comorbidity & \\
\hline & No Comorbidity & $28(40)$ \\
\hline & Hypertension & $24(34.3)$ \\
\hline & CVD & $1(1.4)$ \\
\hline & Cholesterol & $6(8.6)$ \\
\hline & Hypertension + Cholesterol & $5(7.1)$ \\
\hline & Hypertension + Cholesterol + CVD & $3(4.3)$ \\
\hline & Others & $3(4.3)$ \\
\hline 5 & Weight (kg), Mean + SD & $93.70 \pm 3.92$ \\
\hline 6 & BMI $\left(\mathrm{kg} / \mathrm{m}^{2}\right)$, Mean $\pm \mathrm{SD}$ & $33.74 \pm 5.89$ \\
\hline \multirow[t]{2}{*}{7} & Dosage of Liraglutide & \\
\hline & $0.8 \mathrm{mg}$ & $1(1.4)$ \\
\hline
\end{tabular}




\section{Cureus}

\begin{tabular}{|c|c|c|}
\hline & $1.2 \mathrm{mg}$ & $60(85.7)$ \\
\hline & $1.8 \mathrm{mg}$ & $9(12.9)$ \\
\hline \multirow[t]{8}{*}{8} & Medication History & \\
\hline & Liraglutide & $18(25.7)$ \\
\hline & Liraglutide + SGLT2 Inhibitors & $17(25.7)$ \\
\hline & Liraglutide + Sulfonylureas & $7(10.0)$ \\
\hline & Liraglutide + Metformin & $2(2.9)$ \\
\hline & Liraglutide + Metformin + SGLT2 inhibitors & $5(7.1)$ \\
\hline & Liraglutide + Pre-Mix Insulin & $9(12.9)$ \\
\hline & Liraglutide + Long-Acting Insulin & $18(25.7)$ \\
\hline 9 & HbA1C (\%), Mean \pm SD & $9.57 \pm 2.45$ \\
\hline 10 & SBP $(\mathrm{mmHg})$, Mean \pm SD & $136.78 \pm 17.63$ \\
\hline 11 & DBP $(\mathrm{mmHg})$, Mean \pm SD & $88.6 \pm 10.49$ \\
\hline 12 & Blood Urea (mg/dL), Mean \pm SD & $29.33 \pm 11.15$ \\
\hline 13 & Creatinine $(\mathrm{mg} / \mathrm{dL})$, Mean \pm SD & $0.8 \pm 0.22$ \\
\hline \multirow[t]{4}{*}{14} & Diet & \\
\hline & Poor Control & $25(35.7)$ \\
\hline & Moderate Control & $40(57.1)$ \\
\hline & Strict Control & $5(7.1)$ \\
\hline \multirow[t]{4}{*}{15} & Exercise & \\
\hline & No & $42(60.0)$ \\
\hline & Moderate & 27 (38.6) \\
\hline & Strict & $1(1.4)$ \\
\hline
\end{tabular}

\section{TABLE 1: Descriptive analysis of patients on liraglutide at baseline}

SD, standard deviation; CVD, cardiovascular disease; BMI, body mass index; SGLT2, sodium-glucose co-transporter-2; HbA1C, glycated hemoglobin; SBP, systolic blood pressure; DBP, diastolic blood pressure

Table 2 shows the comparison of variables from baseline to follow-up of patients taking liraglutide. The mean difference in weight, BMI, HbA1C, systolic blood pressure, and diastolic blood pressure from baseline to follow-up is $-5.36 \mathrm{~kg},-2.14 \mathrm{~kg} / \mathrm{m}^{2},-1.76 \%,-12.38 \mathrm{mmHg}$, and $5.55 \mathrm{mmHg}$, respectively. When inquired on follow-up, majority of the patients reported having nausea (48\%) as a side effect of liraglutide, and a few reported to experience diarrhea, vomiting, and headache as well. 


\section{Cureus}

\begin{tabular}{|c|c|c|c|c|}
\hline S. No & Variable & At Baseline (Mean) & At 3-6 Months Follow-up (Mean) & Difference (Mean) \\
\hline 1 & Weight (kg) & 93.70 & 88.34 & -5.36 \\
\hline 2 & BMI $\left(\mathrm{kg} / \mathrm{m}^{2}\right)$ & 33.74 & 31.6 & -2.14 \\
\hline \multirow[t]{3}{*}{3} & Blood Pressure & & & \\
\hline & Systolic Blood Pressure (mmHg) & 136.78 & 124.4 & -12.38 \\
\hline & Diastolic Blood Pressure (mmHg) & 88.6 & 83.05 & -5.55 \\
\hline 4 & HbA1C (\%) & 9.57 & 7.81 & -1.76 \\
\hline 5 & Fasting Plasma Glucose (mg/dL) & 213.57 & 124.4 & -89.17 \\
\hline \multirow[t]{8}{*}{6} & Side Effects, n (\%) & & & \\
\hline & Nausea & & $34(48.6)$ & \\
\hline & Diarrhea & & $2(2.9)$ & \\
\hline & Vomiting & & $2(2.9)$ & \\
\hline & Headache & & $1(1.4)$ & \\
\hline & Nausea + Diarrhea & & $1(1.4)$ & \\
\hline & Nausea + Vomiting & & $5(7.1)$ & \\
\hline & Nausea + Headache & & $1(1.4)$ & \\
\hline
\end{tabular}

\section{TABLE 2: Comparison of variables from baseline to follow-up of liraglutide treatment}

$\mathrm{BMI}$, body mass index; HbA1C; glycated hemoglobin

TSQM-9 scores were compared from baseline, and it was found that patients are satisfied with the treatment and its effectiveness $(\mathrm{p}<0.05)$, whereas in terms of convenience, there was no significant difference (Table 3).

\begin{tabular}{|c|c|c|c|}
\hline S. No. & PROs & Baseline & Follow-up \\
\hline 1 & Domain 1: Effectiveness & 50.95 & 71.34 \\
\hline 2 & Domain 2: Convenience & 69.06 & 71.34 \\
\hline 3 & Domain 3: Global Satisfaction & 47.95 & 69.38 \\
\hline
\end{tabular}

TABLE 3: PROs: TSQM-9

PROs, patient-reported outcomes; TSQM-9, Treatment Satisfaction Questionnaire for Medication

Of the patients, 18 were prescribed liraglutide only, 25 liraglutide and oral anti-diabetics (SGLT-2 inhibitors, metformin, sulfonylurea), and 27 liraglutide and insulin. These three groups were assessed individually (Table 4), showing significant results, and collectively no significant changes were observed in terms of glycemic levels $(p>0.05)$ but changes in weight were identified in the insulin group as well (Table 5). Figure 1 shows the difference in baseline values comparing all three therapy groups. 


\section{Cureus}

\begin{tabular}{|c|c|c|c|c|c|}
\hline \multirow[t]{2}{*}{ S. No } & .Variables & Pre-treatment (Mean) & Post-treatment (Mean) & Difference & p-Value \\
\hline & \multicolumn{5}{|c|}{ Group 1: Liraglutide as Monotherapy $(n=18)$} \\
\hline 1 & Weight & 91.77 & 85.38 & 6.39 & $<0.001$ \\
\hline 2 & BMI & 32.85 & 30.26 & 2.59 & $<0.001$ \\
\hline 3 & HbA1C & 8.58 & 6.79 & 1.79 & $<0.001$ \\
\hline 4 & Fasting Glucose Levels & 189.72 & 118.22 & 71.5 & $<0.001$ \\
\hline 5 & Systolic Blood Pressure & 130.83 & 117.77 & 13.06 & 0.001 \\
\hline \multirow[t]{2}{*}{6} & Diastolic Blood Pressure & 88.61 & 81.11 & 7.50 & $<0.001$ \\
\hline & \multicolumn{5}{|c|}{ Group 2: Liraglutide with OADs (n=25) } \\
\hline 1 & Weight & 93.88 & 89.09 & 4.79 & $<0.001$ \\
\hline 2 & BMI & 33.36 & 31.58 & 1.78 & $<0.001$ \\
\hline 3 & HbA1C & 9.73 & 7.98 & 1.75 & $<0.001$ \\
\hline 4 & Fasting Glucose Levels & 202.92 & 124.40 & 78.52 & $<0.001$ \\
\hline 5 & Systolic Blood Pressure & 140.56 & 126.40 & 14.16 & $<0.001$ \\
\hline \multirow[t]{2}{*}{6} & Diastolic Blood Pressure & 89.52 & 83.84 & 5.68 & 0.008 \\
\hline & \multicolumn{5}{|c|}{ Group 3: Liraglutide with Insulin ( $\mathrm{n=27}$ ) } \\
\hline 1 & Weight & 95.00 & 89.60 & 5.4 & $<0.001$ \\
\hline 2 & BMI & 34.78 & 32.70 & 2.08 & $<0.001$ \\
\hline 3 & HbA1C & 10.08 & 8.32 & 1.76 & $<0.001$ \\
\hline 4 & Fasting Glucose Levels & 239.25 & 143.03 & 96.22 & $<0.001$ \\
\hline 5 & Systolic Blood Pressure & 137.22 & 126.29 & 10.93 & $<0.001$ \\
\hline 6 & Diastolic Blood Pressure & 87.77 & 83.70 & 4.07 & 0.068 \\
\hline
\end{tabular}

TABLE 4: Comparison of Liraglutide with OADs and Insulin

BMI, body mass index; HbA1C, glycated hemoglobin; OADs, oral anti-diabetics 


\section{Cureus}

\begin{tabular}{|c|c|c|c|}
\hline S. No. & Variable & Variance & Significance \\
\hline \multirow{2}{*}{1} & \multirow{2}{*}{ Weight } & 0.008 & \multirow{2}{*}{0.997} \\
\hline & & 2.349 & \\
\hline \multirow{2}{*}{2} & \multirow{2}{*}{ BMI } & 13.523 & \multirow{2}{*}{0.415} \\
\hline & & 15.188 & \\
\hline \multirow{2}{*}{3} & \multirow{2}{*}{$\mathrm{HbA1C}$} & 3.461 & \multirow{2}{*}{0.133} \\
\hline & & 1.664 & \\
\hline \multirow[b]{2}{*}{4} & \multirow{2}{*}{ Fasting Glucose Levels } & 3790.982 & \multirow{2}{*}{0.220} \\
\hline & & 2450.111 & \\
\hline \multirow{2}{*}{5} & \multirow{2}{*}{ Systolic Blood Pressure } & 70.093 & \multirow{2}{*}{0.702} \\
\hline & & 197.196 & \\
\hline \multirow[b]{2}{*}{6} & \multirow{2}{*}{ Diastolic Blood Pressure } & 63.826 & \multirow{2}{*}{0.503} \\
\hline & & 91.907 & \\
\hline
\end{tabular}

TABLE 5: Comparison of three liraglutide groups using one-way ANOVA

BMI, body mass index; HbA1C, glycated hemoglobin; ANOVA, analysis of variance

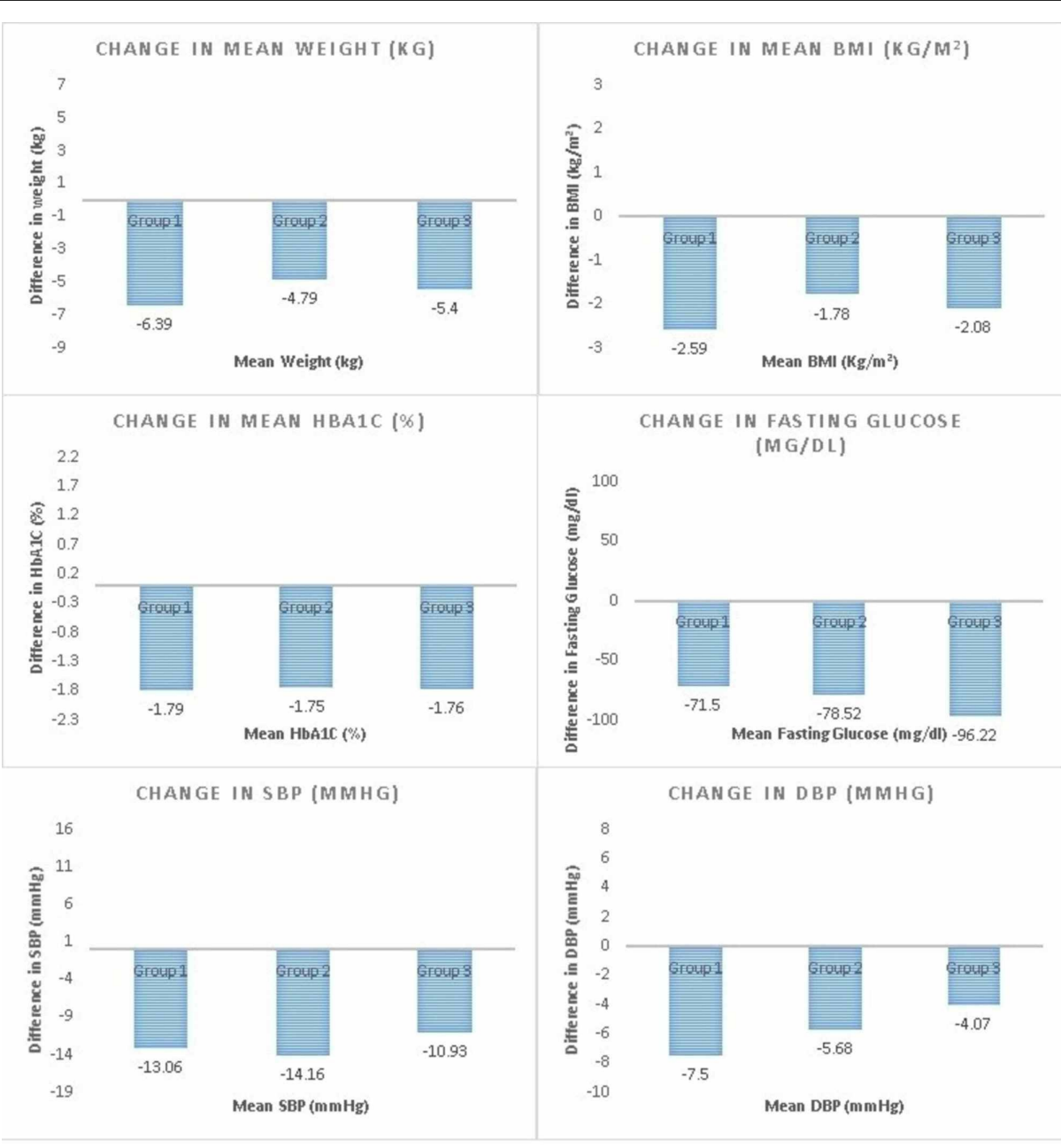

FIGURE 1: Mean changes in baseline characteristics in different groups 


\section{Discussion}

This study shows that liraglutide is beneficial in reducing weight, BMI, HbA1C levels, fasting blood glucose, and blood pressure in Pakistani patients with T2DM. Liraglutide has been shown in LEAD (Liraglutide Effect and Action in Diabetes) trials to improve glycemic control, with HbA1c reduction of up to $0.8-1.6 \%$. We also observed a significant reduction in HbA1C levels similar to the outcome of LEAD trials [9-13]. In Italy, a cohort of 400 patients was prescribed with liraglutide and showed a significant positive determinant for HbA1c reduction after a follow-up of 12 months [14]. Another study reported HbA1C levels decreased from $7.9 \pm 0.9 \%$ at baseline to $7.0 \pm 0.7 \%$ after five years of liraglutide treatment [15]. Fasting plasma glucose (FPG) was also reported to have significant reduction. There was evidence of a dose-dependent impact of liraglutide on FPG, which was more markedly decreased with the $1.8 \mathrm{mg}$ dose than with the $1.2 \mathrm{mg}$ dose [16]. Another study showed a significant reduction in patients’ FPG after 28 weeks of liraglutide use [17].

Incretin-based treatments can produce weight loss in contrast to many alternative treatment plans that often lead to weight gain. In our study, liraglutide also showed its efficacy in weight and BMI reduction when used as a monotherapy or in combination with other oral hypoglycemic agents and insulin. The mean weight and BMI reduction at follow-up from baseline in our study were $5.36 \mathrm{~kg}$ and $2.14 \mathrm{~kg} / \mathrm{m} 2$, respectively. As reported in the LEAD-4 trial, a considerable and sustained weight loss with liraglutide monotherapy was stated in contrast with glimepiride. Weight reduction with liraglutide monotherapy happened usually within the first 16 weeks but was then sustained [12]. Weight loss with liraglutide tends to be dose-dependent both in monotherapy and combination regimens. Throughout the LEAD trials, an analysis of change in body weight stratified through BMI ( $\geqslant 30 \mathrm{vs.}<30 \mathrm{~kg} / \mathrm{m} 2$ ) established that there has been a greater weight reduction in patients with high BMIs [9-13]. Our study shows similar results.

Liraglutide treatment was associated with reduced systolic and diastolic blood pressure. We observed that the mean systolic and diastolic blood pressure reduction at follow-up from baseline in our study was 12.38 $\mathrm{mmHg}$ and $5.55 \mathrm{mmHg}$, respectively. Similar results were observed in our three different therapy groups. These differences in blood pressure were much higher in our study than reported in LEAD studies that showed a reduction of systolic blood pressure between 2.7 and $2.9 \mathrm{mmHg}$ [9-13]. Another randomized controlled trial analyzed the effect of liraglutide on blood pressure and compared it with placebo, showing a reduction in systolic blood pressure by $-5.73 \mathrm{mmHg}$ and a neutral effect on diastolic blood pressure by a mean difference of $-1.42 \mathrm{mmHg}$ [18]. Interestingly, a real-world evidence study from India showed a systolic blood pressure reduction of $9.7 \mathrm{mmHg}$, which was similar to that in our study [19]. We propose that this greater reduction in blood pressure shown in our study was due to greater weight loss $(5.36 \mathrm{~kg})$ seen in our cohort due to liraglutide.

In addition to glycemic and metabolic benefits, liraglutide is well known for gastrointestinal side effects including nausea, vomiting, and diarrhea [20]. Most (48\%) of the patients in our study also reported nausea. Despite this, it was important to note that our study cohort has shown a high adherence rate to liraglutide, with $55 \%$ showing high adherence and $41 \%$ medium adherence as per the MGL Adherence Scale. Also, the treatment satisfaction rate in our cohort was high with TSQM-9, showing an increase in effectiveness from baseline 50.95 to 71.34 and global satisfaction from 47.95 to 69.38 . However, convenience remains unchanged from 69.06 to 71.34 . We, therefore, propose that though liraglutide treatment leads to increase treatment satisfaction and hence a high adherence rate, convenience does not improve due to liraglutide given as a subcutaneous injection once daily.

\section{Strengths and Limitations}

This is one of the few studies that not only evaluate the safety and effectiveness of liraglutide in the Pakistani population but also look at treatment satisfaction and adherence rates. Use of liraglutide has raised the level of treatment satisfaction through better glycemic control and had efficacious treatment adherence. However, the study possesses some limitations being an observational cohort study. Firstly, as data were collected retrospectively, the interval between HbA1c measurements was not constant. Secondly, patient records did not mention whether insulin doses were reduced after initiating liraglutide. Thirdly, as this study analyzed patients treated with liraglutide for a short period, it was not possible to predict therapeutic persistence. Fourthly, although commending BMI and HbA1C levels were reported at follow-up, several confounders such as diet and physical activity could not be monitored in a retrospective study. Lastly, due to the shorter duration of this study, we could not evaluate the cardiovascular and renal benefit of liraglutide in our population. Prospective studies are required to further evaluate the safety, effectiveness, and patient satisfaction of liraglutide, comparing multiple useful consequences of liraglutide in patients with cardiovascular risks and baseline albuminuria and thus evaluating the new cardio defensive and renal protecting role of this drug in South Asian population.

\section{Conclusions}

Our study has demonstrated the effectiveness of liraglutide as a monotherapy or combination therapy in the 
Pakistani population. Liraglutide provided $\mathrm{HbA} 1 \mathrm{c}$ reductions in addition to rapid and sustained reductions in FPG, weight loss, and systolic blood pressure. This is associated with high treatment satisfaction rate and adherence rate. Thus, liraglutide remains an effective though expensive treatment option in a country like Pakistan.

\section{Additional Information \\ Disclosures}

Human subjects: Consent was obtained by all participants in this study. Shifa International Hospitals Ltd issued approval 1153-429-2018. Please note that with reference to your study entitled as "To study the effect of liraglutide on glucose levels, weight reduction, and QOL in type II diabetic patients" has been reviewed by the IRB/EC. The IRB \& Ethics Committee is pleased to approve the study. . Animal subjects: All authors have confirmed that this study did not involve animal subjects or tissue. Conflicts of interest: In compliance with the ICMJE uniform disclosure form, all authors declare the following: Payment/services info: Editorial assistance provided by Peak Medical Services and financially funded by Novo Nordisk. The authors take full responsibility for the content and conclusions stated in this manuscript. Novo Nordisk neither influenced the content of this publication nor was it involved in the study design, data collection, analysis, interpretation or review. Financial relationships: All authors have declared that they have no financial relationships at present or within the previous three years with any organizations that might have an interest in the submitted work. Other relationships: All authors have declared that there are no other relationships or activities that could appear to have influenced the submitted work.

\section{References}

1. Steven P Marso, Gilbert H Daniels, Kirstine Brown al.: Liraglutide and cardiovascular outcomes in type 2 diabetes. N Engl J Med. 2016, 375:311-22. 10.1056/NEJMoa1603827

2. Ryan D, Acosta A: GLP-1 receptor agonists: nonglycemic clinical effects in weight loss and beyond . Obesity (Silver Spring). 2015, 23:1119-1129. 10.1002/oby.21107

3. Advani A, Bugyei-Twum A, Connelly KA: Cardiovascular effects of incretins in diabetes . Can J Diabetes. 2013, 37:309-314. 10.1016/j.jcjd.2013.06.010

4. Thompson PL, Davis TME: Cardiovascular effects of glucose-lowering therapies for type 2 diabetes: new drugs in perspective. Clin Ther. 2017, 39:1012-1025. 10.1016/j.clinthera.2016.10.008.

5. Rashid MO, Jabeen S, Khoja A, Islam N: Efficacy of liraglutide in clinical practice: single centre experience . Pak J Med Sci. 2020, 36:432-437. 10.12669/pjms.36.3.358

6. Kaur P, Mishra SK, Mithal A, Saxena M, Makkar A, Sharma P: Clinical experience with liraglutide in 196 patients with type 2 diabetes from a tertiary care center in India. Indian J Endocrinol Metab. 2014, 18:77-82. 10.4103/2230-8210.126572

7. Atkinson MJ, Sinha A, Hass SL, Colman SS, Kumar RN, Brod M, Rowland CR: Validation of a general measure of treatment satisfaction, the Treatment Satisfaction Questionnaire for Medication (TSQM), using a national panel study of chronic disease. Health Qual Life Outcomes. 2004, 2:12. 10.1186/1477-7525-2-12

8. Beyhaghi H, Reeve BB, Rodgers JE, Stearns SC: Psychometric properties of the four-item Morisky Green Levine Medication Adherence Scale among Atherosclerosis Risk in Communities (ARIC) study participants. Value Health. 2016, 19:996-1001. 10.1016/j.jval.2016.07.001

9. Garber A, Henry R, Ratner R, et al.: Liraglutide versus glimepiride monotherapy for type 2 diabetes (LEAD-3 Mono): a randomised, 52-week, phase III, double-blind, parallel-treatment trial. Lancet. 2009, 373:473-481. 10.1016/S0140-6736(08)61246-5

10. Marre M, Shaw J, Brändle M, et al.: Liraglutide, a once-daily human GLP-1 analogue, added to a sulphonylurea over 26 weeks produces greater improvements in glycaemic and weight control compared with adding rosiglitazone or placebo in subjects with Type 2 diabetes (LEAD-1 SU). Diabet Med. 2009, 26:268-278. 10.1111/j.1464-5491.2009.02666.x

11. Nauck M, Frid A, Hermansen K, et al.: Efficacy and safety comparison of liraglutide, glimepiride, and placebo, all in combination with metformin, in type 2 diabetes: the LEAD (Liraglutide effect and action in diabetes)-2 study. Diabetes Care. 2009, 32:84-90. 10.2337/dc08-1355

12. Zinman B, Gerich J, Buse JB, et al.: Efficacy and safety of the human glucagon-like peptide-1 analog liraglutide in combination with metformin and thiazolidinedione in patients with type 2 diabetes (LEAD- 4 Met+TZD). Diabetes Care. 2009, 32:1224-1230. 10.2337/dc08-2124

13. Russell-Jones D, Vaag A, Schmitz O, et al.: Liraglutide vs insulin glargine and placebo in combination with metformin and sulfonylurea therapy in type 2 diabetes mellitus (LEAD-5 met+SU): a randomised controlled trial. Diabetologia. 2009, 52:2046-2055. 10.1007/s00125-009-1472-y

14. Lapolla A, Frison V, Bettio M, et al.: Correlation between baseline characteristics and clinical outcomes in a large population of diabetes patients treated with liraglutide in a real-world setting in Italy. Clin Ther. 2015, 37:574-584. 10.1016/i.clinthera.2014.11.015

15. Mirabelli M, Chiefari E, Caroleo P, et al.: Long-term effectiveness of liraglutide for weight management and glycemic control in type 2 diabetes. Int J Environ Res Public Health. 2019, 17:207. 10.3390/ijerph17010207

16. Blonde L, Russell-Jones D: The safety and efficacy of liraglutide with or without oral antidiabetic drug therapy in type 2 diabetes: an overview of the LEAD 1-5 studies. Diabetes Obes Metab. 2009, 11:26-34. 10.1111/j.1463-1326.2009.01075.x

17. Seufert J, Bailey T, Barkholt Christensen S, Nauck MA: Impact of diabetes duration on achieved reductions in glycated haemoglobin, fasting plasma glucose and body weight with liraglutide treatment for up to 28 weeks: a meta-analysis of seven phase III trials. Diabetes Obes Metab. 2016, 18:721-724. 10.1111/dom.12623

18. Liakos A, Lambadiari V, Bargiota A, et al.: Effect of liraglutide on ambulatory blood pressure in patients with hypertension and type 2 diabetes: a randomized, double-blind, placebo-controlled trial. Diabetes Obes 


\section{Cureus}

Metab. 2019, 21:517-524. 10.1111/dom.13541

19. Kesavadev J, Shankar A, Gopalakrishnan G, Jothydev S: Efficacy and safety of liraglutide therapy in 195 Indian patients with type 2 diabetes in real world setting. Diabetes Metab Syndr. 2015, 9:30-33.

10.1016/j.dsx.2014.04.034

20. Onoviran OF, Li D, Toombs Smith S, Raji MA.: Effects of glucagon-like peptide 1 receptor agonists on comorbidities in older patients with diabetes mellitus. Ther Adv Chronic Dis. 2019, 10:2040622319862691. $10.1177 / 2040622319862691$ 